\title{
Téoros
}

Revue de recherche en tourisme

\section{Le développement régional dans les politiques québécoises du tourisme}

\section{Jean-Marie Lucas-Girardville}

Volume 5, numéro 1, mars 1986

Tourisme et régions

URI : https://id.erudit.org/iderudit/1080595ar

DOI : https://doi.org/10.7202/1080595ar

Aller au sommaire du numéro

Éditeur(s)

Université du Québec à Montréal

ISSN

0712-8657 (imprimé)

1923-2705 (numérique)

Découvrir la revue

Citer cet article

Lucas-Girardville, J.-M. (1986). Le développement régional dans les politiques québécoises du tourisme. Téoros, 5(1), 36-38. https://doi.org/10.7202/1080595ar d'utilisation que vous pouvez consulter en ligne. 


\title{
Le développement régional dans les politiques québécoises du tourisme
}

\author{
par Jean-Marie Lucas-Girardville*
}

\section{Une très courte histoire \\ Il faut bien l'avouer: le développement tou- ristique régional, au Québec, est encore en gestation.}

Il ne faut pas remonter bien loin, en effet, le cours du temps pour voir surgir les 18 régions touristiques du Québec, puisqu'i] y a dix ans, les intervenants touristiques parlaient peu de développement et encore moins de régions. Le réseau des associations touristiques régionales est né d"une volonté exprimée en 1977 par le ministre Yves Duhaime à l'instigation de son sousministre adjoint au Tourisme, monsieur Robert Prévost, qui m'avait alors confié la tâche d'en implanter les premières structures.

De multiples raisons avaient présidé à une telle orientation: éparpillement des intervenants, multiplicité des demandes de subventions, saupoudrage des investissements, promotion ponctuelle, absence de lignes directrices et d'images de marque en matière de publicité et de développement du produit. On pourrait aisément ajouter à la liste des arguments en faveur d'une régionalisation du tourisme.

D'ailleurs, lors d'une première réunion exploratoire avec des intervenants des milieux régionaux, en septembre 1977, personne ne songea à remettre en question le bien-fondé d'une telle initiative. On émit plutôt des doutes quant à ses chances de succès, surtout à cause des modalités d'implantation et à l'implication concrète du gouvemement dans cette affaire. Ainsi, il faudrait, tôt ou tard, tracer des limites aux régions touristiques, définir des mandats clairs, négocier le financement et, enfin, passer aux actes, c'est-à-dire recruter des membres. Tant du côté de l'État que des régions, il y avait du travail à faire. Quelques réticences semblaient êmaner du milieu: comment convaincre les intervenants locaux qu'à moyen terme, leur intế-

"L'auteuf est coordonnateur du développement regio nal a la Direction de l'analyse et du deveioppement du ministere du Tourisme du Quebec. Cet article ne repreente que l'opinion de l'auteut et non celle du ministere. rêt particulief passe par l'intérết de la région? Comment solliciter d'eux un financement sur lequel on ne peut garantir un retour immédiat? Quelle preuve le gouvernement peut-il fournir qu'il soutiendra un tel réseau?

De son côté, le gouvernement s"interrogeait sur les dangers de ce réseau: combien cela cóutera-t-il? Comment pourra-t-on intégrer ces régions avec le projet de régionalisation supra-municipale? $\mathrm{N}^{4} \mathrm{y}$ a-t-il pas dédoublement avec les mandats des Con. seils régionaux de développement (CRD) et des Conseils régionaux de loisirs (CRL)? Ne va-t-on pas encore créer une autre structure de concertation sans activité opérationnelle? Quelle sera la représentativité de ces structures?

Enfin, en 1979, après avoir mis sur pied et financé des comités provisoires dans la plupart des régions, laissé définir les limites territoriales par le milieu lui-mème, le gouvernement reconnaissait officiellement le réseau d'associations touristiques régionales et ne lui imposait ni structure, ni territoire; il lui reconnaissait le statut d'interlocuteur privilégić en matière de tourisme, lui confiait un rồle opérationnel au niveau de la promotion, un rôle consultatif au niveau du développement et exigeait à court terme un autofinancement de $50 \%$.

Cing ans plus tard, ce taux est d'environ $60 \%$ pour l'ensemble des ATR et il faut avoir assisté au premier gala des Grands prix du tourisme pour constater à quel point il existe désormais un esprit touristique régional: les attitudes et les mentalités ont changé, les querelles de clochers se sont aplanies - je n'ai pas dit qu'elles avaient disparu! - et les intervenants, qu'ils soient publics ou privés, ont à leur disposition des outils techniques leur permettant de se positionner sur l'échiquier touristique national si concurrentiel.

Chacune des régions a construit son ATR selon ses particularites: ainsi, dans les Laurentides c'est l'entreprise privée qui a constitué les fondements de l'ATR, tandis que dans l'Estrie, ce sont les municipalités et qu'au Saguenay/Lac-St-Jean/Chibougamau, ce sont les commissariats industriels.
Ces modèles, bâtis sur les forces du milieu. se sont élargis par la suite pour diversifier le membership et devenir ainsi plus représentatifs

L'une des premières täches des ATR et non la moindre, fut d'acquérir une crédibilité et de prouver leur utilité. Ce fut une excellente stratégie, je crois, de commencer par la promotion: c'est en organisant des campagnes publicitaires rếgionales, en sollicitant de la publicité coop, en participant à des salons, en élaborant des politiques d'accueil régionales et en produisant les brochures touristiques régionales que les ATR devenaient à court terme des plaques toumantes, des fils conducteurs, des tremplins vers les marchés touristiques intra et extra-québécois.

C'est ainsi, également, que l'on prévoit accoutumer les intervenants locaux, ponctuels, à travailler ensemble, à ressentir les

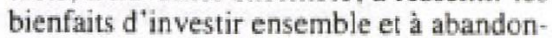
ner peu à peu cette méfiance naturelle réciproque des concurrents voisins, au profit d'une agressivité plus forte vis-à-vis des concurrents plus lointains... En fin de compte, à développer un sentiment d'appartenance régionale!

Les régions touristiques ont donc atteint aujourd hui une certaine maturité: il y a cing ans, les réunions avec les ATR portaient sur des problèmes de survie et de structures. Aujourd "hui, on y parle beaucoup plus de tourisme.

J'ai voulu tracer cet historique pour montrer à quel point, peu à peu, les régions ont évolué et en sont arrivees à un constat évi. dent: dans le toufisme comme dans n'importe quelle activité économiqué, on ne peut structurer la mise en marché d'un produit, on ne peut se préoccuper de la demande régionale, solliciter des marchés, des clientèles sans tôt ou tard se préoccuper du produit que l'on a à vendre, de son implantation. de son évolution. de son image, de sa croissance quantitative et qualitative. En un mol, sans faire du développement. C'est ce qui a amené le ministère du Tourisme. il y a quelques mois, à proposer aux ATR de s'impliquer dans cette activite. Non pas à titre d'investisseurs. 
bien sưr, mais à titre de coordonnateurs, de lieux de concertation des diverses ins. tances régionales.

\section{La position stratégique des ATR}

Mais pourquoi les asociations touristiques? La réponse est biens imple: elles seules peuvent offrir actuellement, dans un cadre ouvert et élargi, une représentativité multi. sectorielle et multi-structurelle. Le cas du tourisme est, en effet, très particulier: il est le seul secteur d'activité économique à impliquer directement ou indirectement une aussi grande variété d'intervenants, puisqu'il ne fait qu'emprunter la plupart du temps à une multiplicité d'activités qui luj sont directement ou indirectement reliées.

Or, le développement touristique, cela $s$ 'organise comme une symphonie. Chacun doit avoir sa partition, chaque musicien son instrument, mais personne ne doit jouer tout seul et à sa façon. Il faut un chef d'orchestre, seul ćlćment capable d'insuffler une mểme atmosphère, de synchroniser les inverventions et d'harmoniser les interprétations.

Il existe dans les régions touristiques des regroupements politiques, administratifs ou associatifs, mais aucun d"entre eux ne peut à lui seul prétendre représenter l'ensemble de l'activité touristique: ce sont les MRC, les Conseils régionaux de la culture ou des loisirs, les Conférences administratives régionales, les Chambres de commerce, etc.

L'énoncé gouvernemental concernant la régionalisation intitulé Le choix des régions proposait des Conseils régionaux de concertation et d'intervention qui $n$ 'ont pas reçu un accueil favorable lors des audiences publiques.

En l'absence d'alternative, le ministère du Tourisme a done proposé aux ATR d'être le lieu de rencontre et d'expression des volontés du milieu dans le domaine de la planification du développement touristique régional.

C'est ainsi que le programme des plans de développement touristique régional fut conçu: gérés administrativement et techniquement par les ATR, ils sont cependant orientés et dirigés par des comités de gestion décisionnels regroupant les forces du milieu: entreprises privées, organismes touristiques de zones, MRC, ministères en régions, conseils consultatifs. Telle est la condition préalable à ce que le plan de développement ne devienne pas le plan de I"ATR ou du ministère, mais bien celui de la région.

En ce sens, c'est une experience-pilote de développement économique régional que le ministère entreprend avec cette vaste opération à l'échellè du Québec. De fait. deux plans sont en consultation. deux autres en gestation, cinq autres viennent de débuter et l'on en prévoit six autres en 1986-87.

En 1987, 15 des 18 régions touristiques auront fait l'objet d'un plan de développement qui servira alors de fil conducteur aux intervenants publics et privés. pour aménager, réglementer, développer ou protéger, investir, aider et orienter l'investissement privé.

Les MRC, dans certains cas, ont émis quelques réticences à s'impliquer dans les comités de gestion des plans de développement et parfois pour des raisons contradictoires: certaines ne se sentent pas prêtes à s'impliquêr dans le développement, ayant déjả beaucoup à faire avec les schémas d'aménagement; d'autres, en revanche, voient dans l'ATR une autorité imposée d'en haut quil leur dira quoi faire et, dans le plan de développement, un carcan dans lequel leus schéma d'aménagement devra s'insérer. Je crois qu'une mise au point $s^{+}$impose à ce sujet: il est clair que les MRC sont des entités politiques ayant une existence légale et que les schémas d"aménagement sont des documents élaborés en vertu de la Loi sur l'amenagement et l'urbanisme, suivant un long processus. De ce fait, les schémas d'aménagement ont force de loi et ont par conséquent prépondérance sur les plans de développement.

Ce sont donc les plans qui devraient considérer les schémas comme une contrainte de base et $s^{2} y$ adapter. D'ailleurs, le gouvernement, en vertu des articles 11 et 16 de la Loi 125 , a déjà formulé des orientations et des avis aux MRC concernant leurs propositions d'aménagement.

I est certain cependant que sur le plan théorique, il eut été souhaitable que les schémas puissent s'élaborer individuellement à partir d'une stratégie de développement touristique commune et concertée par l'ensemble des MRC et autres intervenants de la région. Mais le résultat n'en aurait peut-ètre pas été meilleur, compte tenu du contexte chronologique et politique. On constatera peut-être " "à posteriori" que pour une fois, il aura été préférable de mettre la charrue avant les boeufs!

En bref, il valait mieux que les municipalités s'exercent à la concertation au niveau du schéma et de la MRC, avant de leur imposer une concertation au niveau régional.

Quoi qu'il en soit, les plans de développement arrivent au bon moment, non seulement sur l'échiquier politique régional. mais aussi dans le processus gouvememental qui a entouré la création du ministère du Tourisme. C'est en 1985, en effet, que furent créées la Direction générale du développement et l'équipe ministérielle du développement régional. que fut signée
l'Entente auxiliaire Canada-Québec sur le tourisme, que les 17 ATR existantes se sont enfin regroupées dans un seul organisme et que fut diffusé pour consultation le document d'orientations ministériel intitulé: $\mathbf{L e}$ tourisme au Québec (Bilan et perspectives d'action 1985-88). C'est aussi en 1985 que les contacts ministère du TourismeMRC se sont intensifiés.

\section{Objectifs des plans de développement régional}

Les plans de développement régional poursuivent quatre objectifs principaux

a) produire un document de référence commun à tous les intervenants pour déve. lopper et mettre en marché le produit régional,

b) instaurer des mécanismes nouveaux de concertation en matiêre de développement touristique:

c) faire un lien entre l'offre et la demande touristique;

d) déboucher à moyen terrme sur une stratégie multi-régionale, résultante de l'ensemble des plans.

L'operation plans de développement n'est pas une fin en soi. Ce n'est qu'un tremplin, un préalable nécessaire dont le suivi, ce que j"appelle "I'après -plan" , sera peut-être à la fois la phase la plus importante, mais aussi la plus difficile.

Dès à présent, une réflexion conjointe

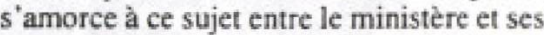
partenaires régionaux. Quelques questions s'imposent: faut-il une structure pour faire le suivi et si oui, laquelle? Comment peuton assurer l'influence la plus grande $d u$ plan sur le développement régional? Doitil être mis à jour? Quelle sera son espérance de vie? Comment obtenir sa diffusion et sa compréhension maximales auprès des investisseurs potentiels? De quels compléments a-t-il besoin? Comment l'harmoniser avec ceux des autres régions? Comment en faire un stimulant plutôt qu'une contrainte? Là encore, les réponses ne seront pas universelles, elles devront s'adapter au contexte régional.

Mais dans toute la question du developpement touristique régional, il $y$ a deux points sur lesquels on $n$ insistera jamais assez: l'effet de synergie et l'approche-client, qui sont d'ailleurs interreliés.

L'effet de synergie c'est l'effet moteur. Lorsqu'on développe une région touristique, on doit faire des choix, s'entendre sur une ou des priorités, privilégier des territoires par rapport à d'autres. On ne développe pas une région "tous azimuths" sous prétexte que chacun a droit à sa part des retombées du tourisme et considère que "c est beau chez nous, on a des lacs. des. rivières et des montagnes". La question 
n'est pas tant de savoir en effet ce qu'on a de bien, mais ce qu on a de mienr que les autres. L'effet de synergie, c'est donc miser sur ce qu'on a de mieux et d'y consacrer tous ses efforts. Par un phénomène de "tache d'huile", le developpement finira à moyen terme par se répercuter sur les póles secondaires. Mais de telles stratégies exigent une maturité politicoéconomiqué que certaines régions ne sont pas encore en mesure d'exercer.

Le meilleur exemple. sinon le seul, est sans doute celui de l'Estrie qui a su faire de Magog-Orford sa priorité. consciente que l'ensemble de la région en récoltera les bienfaits.

L'approche-client rejoint aussi cette attitude. On a beaucoup tendance. dans les régions, à vouloit developper pour soiméme, à s' interroger sur le type de développement que l'on voudrait ou qu"on ne voudrait pas. Il ne faut pas oublier cependant. que le touriste, lui, est le décideur final puisque cest lui qui choisira sa destination de voyage. Il aura le dernier mot et, à ce titre, c'est au type de développement qu'il veut, lui, qu'il faut d'abord penser. C'est une des raisons pour lesquelles les études de marché régionales font partic intégrante des plans de developpement et permettront de formuler des stratégiesmarketing régionales.
Je ne peux m"empecher de paraphraser John F. Kennedy en disant: "Pensez à ce que vous pouvez faire pour le tourisme avant de vous demander ce qu'il peut faire pour vous!"

Ce que je viens d'affirmer mériterait évidemment d'être nuancé, mais je voulais simplement en souligner l'essentiel.

Sur le plan des structures. le ministère du Tourisme ne posseddant pas de bureaux régionaux calqués sur les régions administratives, il lui est beaucoup plus facile de structurer ses secteurs d'intervention en fonction des régions touristiques.

C'est pourquoi l'équipe du développement régional, qui compte une dizaine de professionnels, fonctionne par responsabilité d'ATR, cest-à-dire que chacune des régions touristiques n"a qu "un seul interlocuteur en matière de développement. Ce groupe fonctionne comme une équipe volante, ce qui lui confere de la polyvalence et de la souplesse. Cette façon de fonctionner semble, pour le moment, donner toute satisfaction aux ATR.

Cette equipe gère non seulement les plans de développement et les études de faisabilité, mais formule les avis aux MRC dans le cadre de la Loi 125 et procède à l'analyse d'une multitude de projets publics ou d'organismes sans but lucratif, en plus d collaborer à la preparation des conférence socio-économiques régionales.

Elle doit aussi collaborer avec les autre ministeres présents dans le developpemen touristique régional, notamment I $\mathrm{OPDQ}$ le MLCP et les ministères des Affaires cul turelles. de l'Environnement, des Trans ports et des Affaires municipales.

Le développement régional est une activitt de plus en plus présente à cause de la ten dance à la décentralisation et du fait que $k$ sentiment d"appartenance à une commu. nauté économique se fait avant tout sur lis base d'une appartenance territoriale à unt communauté géographique

Ce principe fondamental ne doil pas empecher cependant de s'orienter aussi vers une approche sectorielle, sur la base d'une communauté d'activités, indépendante du territoire. Ces deux visions sont étroitement complémentaires puisque, dans le produit touristique québecois. comme dans tout autre, la somme des secteurs est égale à la somme des régions et réciproquement. $f$

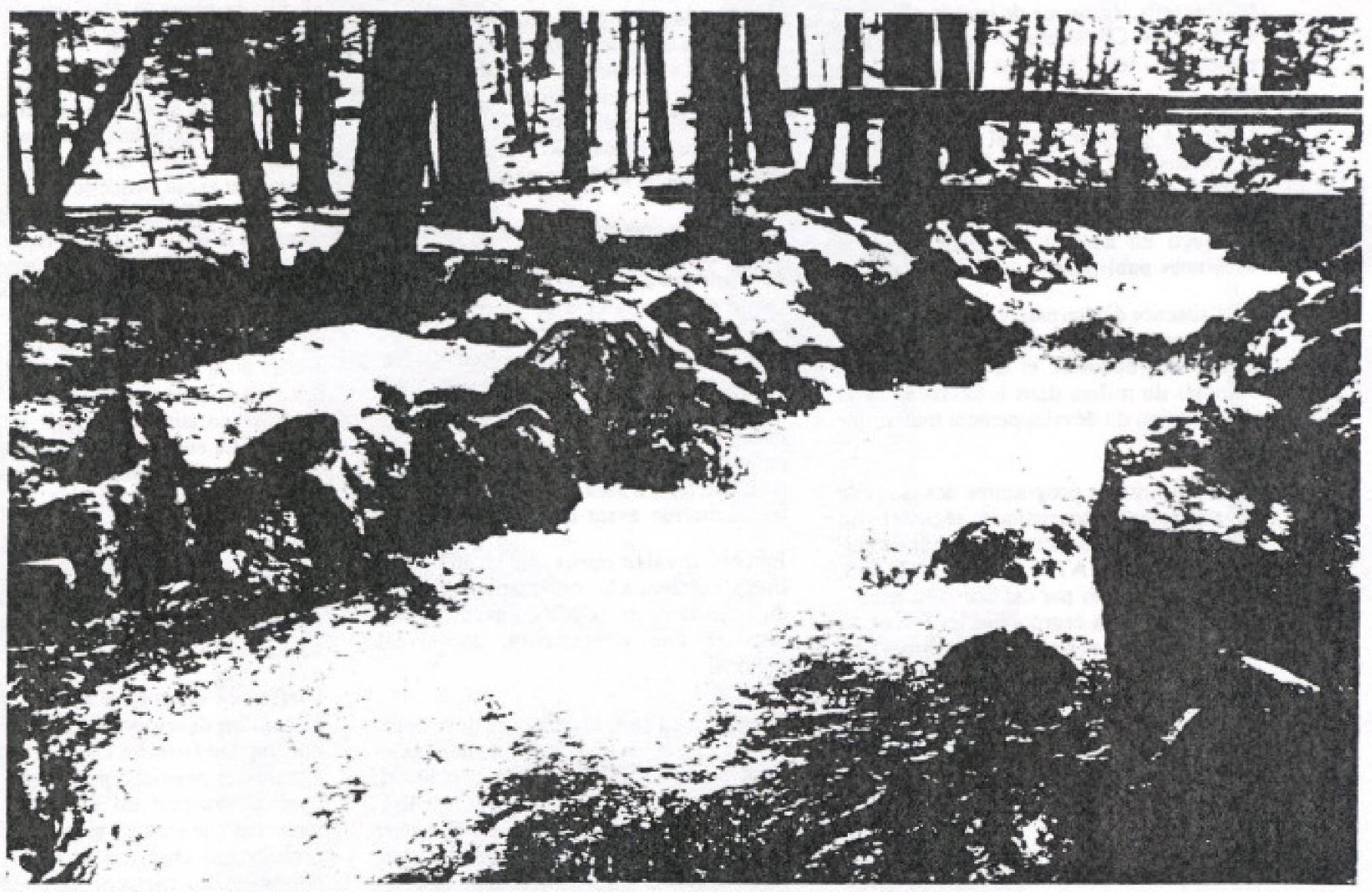

C'est beau chez nous, on a des lacs, des rivieres et des montegnes: la question n'est pas de savoir ce qu'on a de bien, mais ce qu'on a de mieux que les autres. 